\title{
EL RÉGIMEN HIDROLÓGICO DEL RÍO CINCA A FINALES DEL SIGLO XIX*
}

\author{
R. Galván Plaza
}

Confederación Hidrográfica del Ebro, Ministerio de Medio Ambiente, P. Sagasta, 2426, 50071 Zaragoza, rgalvan@chebro.es

\begin{abstract}
Resumen: El Proyecto de Riegos del Alto Aragón de 1911 recoge en su Anejo 6 varias tablas de caudales de diferentes ríos, a los cuales se cita como oficiales de la extinta División Hidráulica del Ebro, entre los que se encuentra una serie de más de 9 años del río Cinca en su desembocadura, correspondiente al periodo 1881-1894. Estos datos hoy olvidados resultan ser una las mediciones sistemáticas más antiguas de las que podemos tener conocimiento, por lo que se convierten en un documento inestimable para la descripción del régimen hidrológico de uno de los ríos principales de la cuenca del Ebro en aquella época y de la evolución sufrida, tanto por circunstancias naturales como por su intenso aprovechamiento humano durante el siglo XX. Además, su recuperación alberga indudable interés para estudios de evolución climática y morfológica. Todo ello a pesar de que del análisis de los datos se desprende una significativa falta de precisión de medida.
\end{abstract}

Palabras clave: río Cinca, régimen hidrológico, caudales, evolución hidrológica, cuenca del Ebro

\begin{abstract}
The 1911 "Riegos del Alto Aragón" project, includes in its $6^{\text {th }}$ annex several stream flow data from different rivers, which are quoted as official data from the extinct "División Hidráulica del Ebro". Among them there is a 9-year period of flow rate from the Cinca river at the confluence site with the Ebro river, corresponding to the period 1881-1894. These forgotten data, are one of the most old systematic flow gauging we know in the Ebro basin, so it is an inestimable document for the description of the hydrologic regime of one of its main tributaries in those far years and its evolution to its present status, both for natural causes and for the intense human development during the XX century. Besides, bringing to life these data has interest for future studies of climatic and morphologic evolution, in spite of the lack of accuracy that the data analysis has proved.
\end{abstract}

* Recibido: 25-10-2005. Aceptado: 30-3-2006. 
Key words: Cinca river, hydrologic regime, flow, hydrologic evolution, Ebro basin

\section{Introducción y antecedentes}

Los datos de caudales aforados en los ríos de la cuenca del Ebro, al igual que los de toda España, comenzaron a ser publicados en 1913 por parte del entonces denominado Servicio Central Hidráulico, a partir de los valores suministrados por las respectivas Divisiones Hidráulicas, la primera administración periférica española de aguas, creada en 1865 según el concepto de cuenca hidrográfica. Por esta razón, las series de aforos existentes, consideradas hoy en día como oficiales y utilizadas como tales en los correspondientes estudios hidrológicos tienen, como pronto, su fecha de inicio en el 1 de enero de $1913^{1}$.

Sin embargo, antes de que comenzara la publicación regular de los datos de aforo, la División Hidráulica del Ebro ya realizaba aforos de caudales de forma sistemática en diferentes ríos de la cuenca, desde fechas tan tempranas como 1881 e incluso anteriores (Mateu Bellés, 2003). Desgraciadamente, todo parece indicar que los registros originales de esta información han desaparecido, al menos de los archivos de la Confederación Hidrográfica del Ebro, a pesar de las provisiones que para su conservación prescribía la Instrucción de Aforos de 1911, que a la vez establecía el sistema nacional para la recopilación y publicación de los datos de caudales de forma homogénea, de lo que sería fruto la primera publicación de 1913.

Por todo ello, reviste gran interés que en el Proyecto de Riegos del Alto Aragón de 1911, se transcribieran en su Anejo 6, unas series de caudales diarios e hidrogramas en varios puntos de los ríos que habían de quedar integrados en el sistema de Riegos del Alto Aragón (sistema que hoy en día cuenta con 120.000 ha de regadío), de los cuales se dice que "son los (caudales) oficiales proporcionados por la División Hidráulica del Ebro". En concreto se recopilan datos de caudal de los ríos Ésera, Gállego, Cinca, Ara y Guatizalema, de distintos periodos temporales (tabla 1).

De todas ellas, la serie más larga, antigua y completa, es la correspondiente al río Cinca en el ex-monasterio de Escarpe, que cubre el periodo 1881-1894. Esta estación se ubica en un punto muy próximo a la desembocadura de este río en el Segre, en las cercanías de un monasterio en aquella época ya inhabitado.

\footnotetext{
1 Aunque debieran iniciarse en 1912, en el caso del Ebro en la primera publicación no se llegan a incluir las tablas de caudales y sólo son dibujados los hidrogramas resultantes correspondientes a 1912.
} 
Tabla 1. Puntos de medida de caudales y periodo de las series en el Proyecto de Riegos del Alto Aragón de 1911.

\begin{tabular}{|lc|}
\hline Denominación puntos de aforo & Periodo \\
\hline Ésera en toma del Canal de Aragón y Cataluña & $1906-1914$ \\
Gállego en puente de Santa Isabel & $1881-1889$ \\
Gállego en barranco de la Junquera & $1897-1898$ \\
Gállego en presa y canal de las "Fuerzas motrices del Gállego" & $1910-1911$ \\
Cinca en el puente del El Grado & $1910-1911$ \\
Cinca en ex-monasterio de Escarpe & $1881-1894$ \\
Ara en puente de Jánovas & 1888 \\
Guatizalema en La Almunia del Romeral & $1907-1910$ \\
\hline
\end{tabular}

Fuente: Proyecto citado.

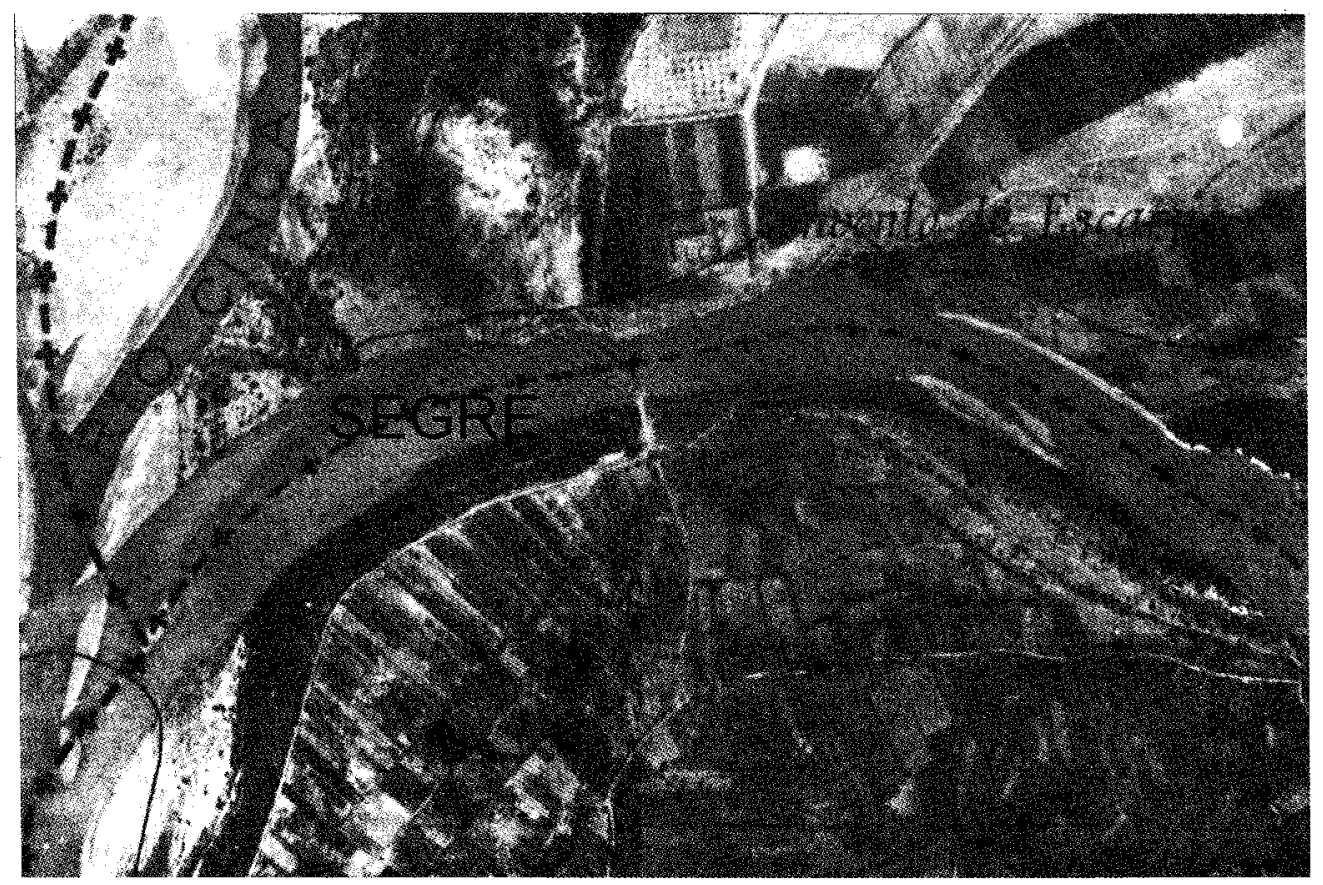

Figura 1. Desembocadura del Cinca en el Segre. Fotoplano del vuelo de Confederación Sindical Hidrográfica del Ebro, 1927.

Fuente: Confederación Hidrográfica del Ebro. 


\section{Descripción}

Esta serie del Cinca en desembocadura contiene 3.840 datos de caudal diario, en teoría caudal medio diario, pero es más que probable que se trate de una simple lectura diaria de escala, a la que se suponga constante durante el día (Figura 2). Estos datos diarios permiten disponer de 9 años hidrológicos completos, más otros 18 meses que se distribuyen en otros 3 años. Además, habiéndose realizado la revisión y digitalización de los datos, no se desprenden incoherencias o errores en la lectura o transcripción significativos (Figura 3. A y B); tan solo se han detectado algunos errores aislados de transcripción y dos meses cuyos valores resultan idénticos día a día (julio y agosto de 1890), seguramente un error en el traslado de los datos, ya sea en el original o en la copia del proyecto.

Dicha serie resulta lo suficientemente extensa y completa, el periodo reflejado tan lejano, y su ubicación tan singular como para permitir la descripción del régimen hidrológico general de la cuenca del Cinca en una época en que no puede sino mostrársenos con unas características de naturalidad hoy desconocida, después de más de un siglo de desarrollo hidráulico. También la serie del Gállego en Santa Isabel para el periodo 1881-89 resulta especialmente interesante, pero aparte de albergar mayor

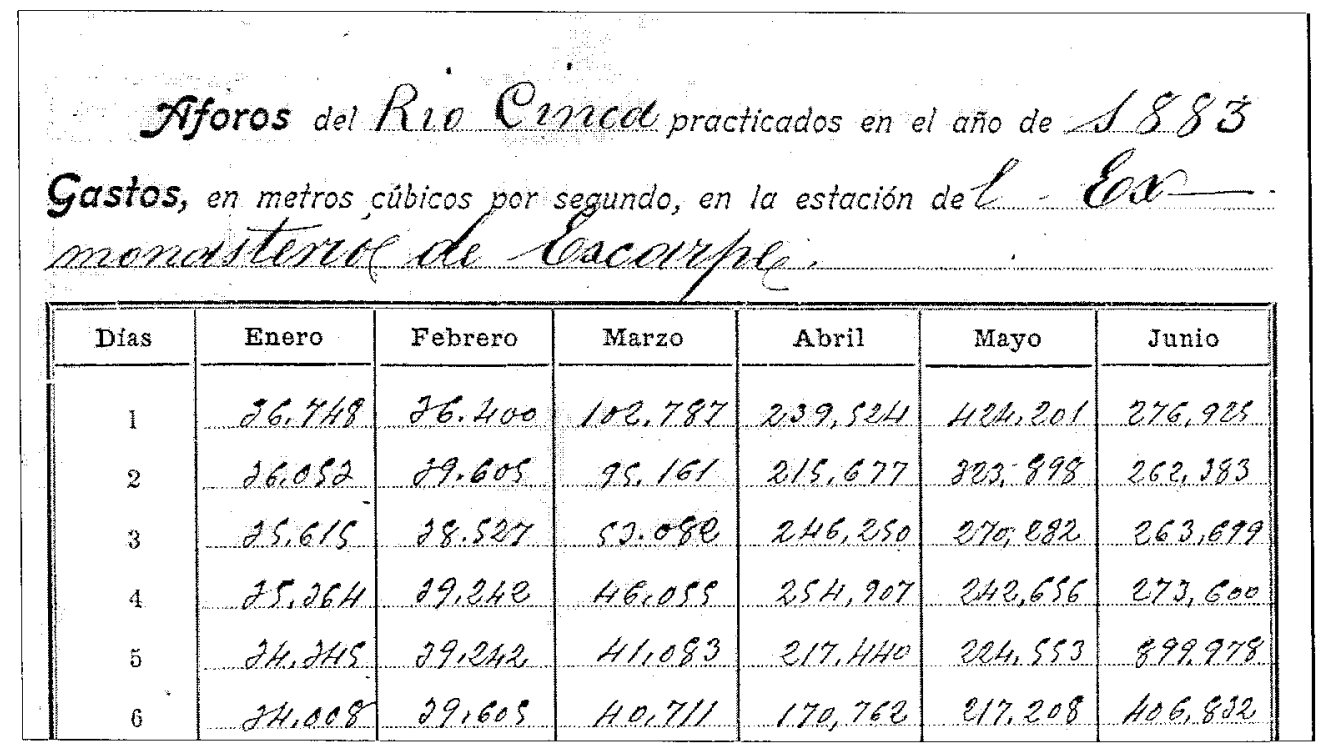

Figura 2. Fragmento de la transcripción de los datos de aforo en el proyecto de Riegos del Alto Aragón.

Fuente: Proyecto citado. 

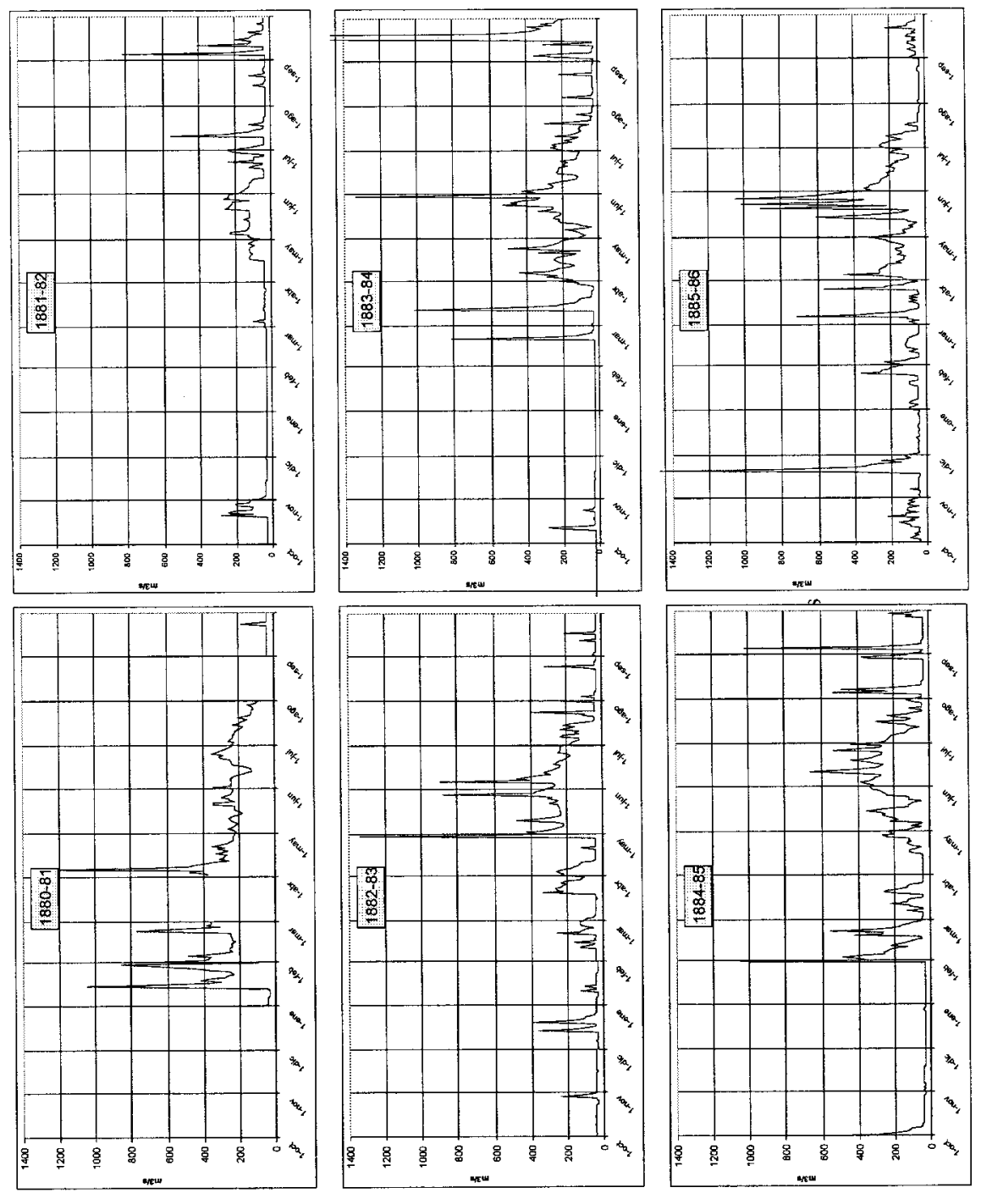

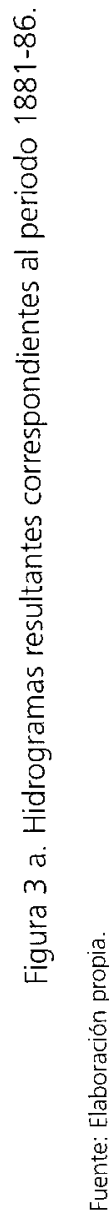



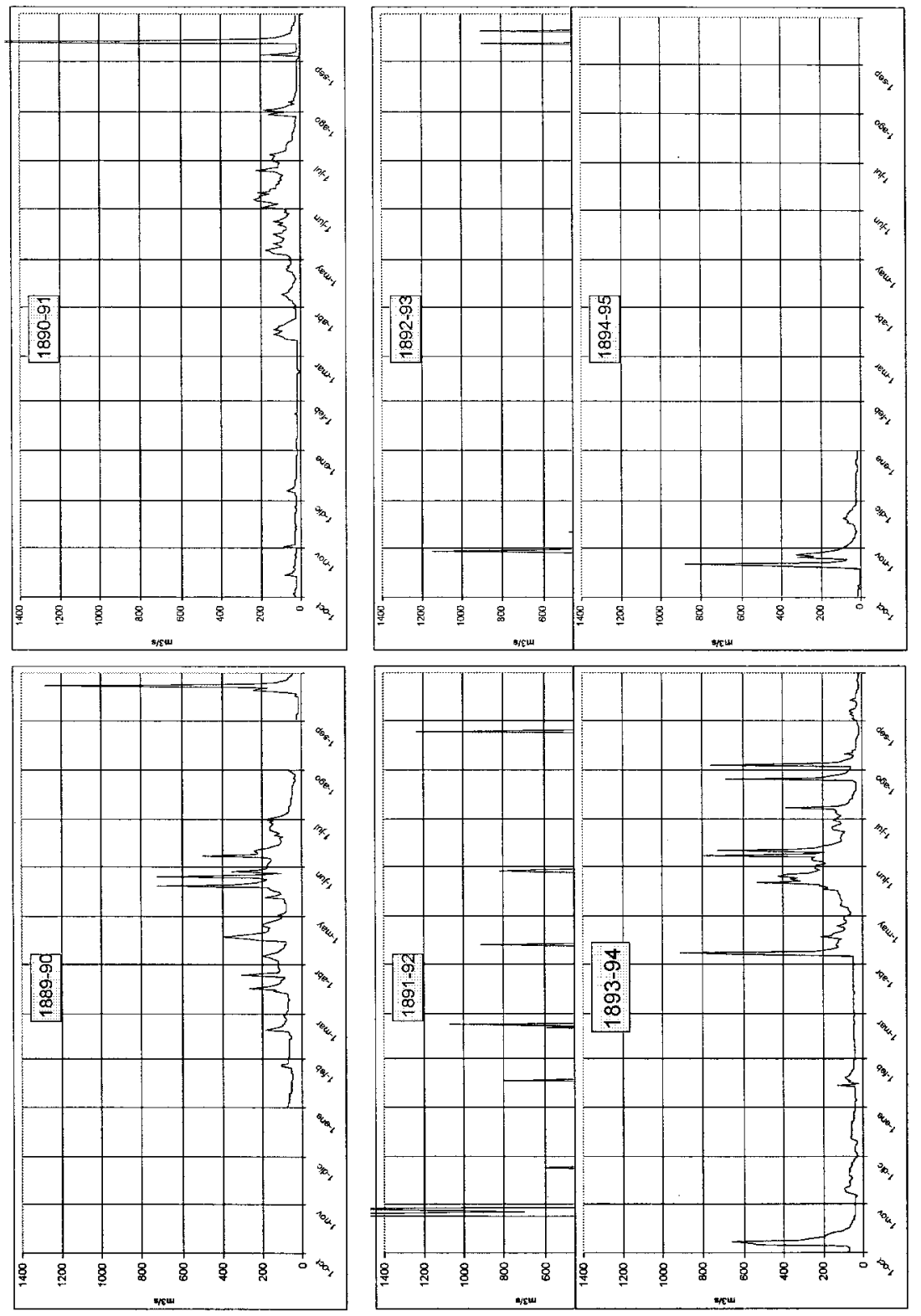
número de huecos en los datos diarios, pudiéndose completar caudales para sólo 3 años completos y otros 28 meses, el propio proyecto afirma que presenta incoherencias difícilmente explicables, lo cual ha podido ser constatado en su revisión.

La serie del Cinca presenta dos etapas diferenciadas; la primera alcanza hasta septiembre de 1886 y después de la última casilla rellena, figura la anotación "cambio de sección", no volviéndose a medir hasta enero de 1889 y finalizando definitivamente en diciembre de 1895, no habiendo registros posteriores a esta fecha y habiendo desaparecido cualquier referencia a esta estación en la primera publicación de datos de aforo de 1913, salvo en el mapa general de la cuenca que la precede, en el cual, en el punto donde debiera quedar situada esta estación encontramos una de código 17', pero ni se describe ni se recogen ningún tipo de datos.

Como se verá más adelante, el cambio de sección que se anota resulta relevante, al menos en lo que se refiere a los caudales mínimos, pues estos no bajan de $20 \mathrm{~m} 3 / \mathrm{s}$ en la primera parte de la serie, mientras que en la segunda se sitúan sobre los 10 $\mathrm{m} 3 / \mathrm{s}$, sin que pueda explicarse este cambio porque existan diferentes comportamientos de las aportaciones anuales o mensuales.

Esta circunstancia nos sirve para apercibirnos y no otorgar a estos datos de caudales de una precisión que desconocemos si tienen. Además el hecho de que posteriormente se considerara su sustitución por la vigente estación de aforos de la Confederación Hidrográfica del Ebro, n $n^{\circ} 17$ Cinca en Fraga, puede ser reflejo de su falta de idoneidad como estación de medida, aunque también pudo deberse a modificaciones naturales en el cauce que imposibilitaran seguir midiendo en el mismo lugar; por otro lado el proyecto de Riegos del Alto Aragón no despeja ninguna incertidumbre, puesto que para sus cálculos se centra en el año que consideran más desfavorable 1908-09, utilizando las series del "Ésera en la toma del canal de Aragón y Cataluña" y del "Cinca en el puente de El Grado".

\section{Análisis y resultados}

A pesar de todas estas cautelas, se pueden extraer bastantes elementos interesantes del estudio de estos datos. Una primera caracterización a partir de la serie de caudales disponible nos presenta un régimen hidrológico a finales del XIX significativamente más nival, con dos mínimos bien marcados en invierno y verano de similares magnitudes (Figura 4.A). El régimen es en realidad bastante similar en su tipología al de la primera mitad del siglo XX, según se desprende de la comparación con los datos procedentes de la estación de aforos $\mathrm{n}^{\circ} 17$ Cinca en Fraga (Figura 4.B), aunque la nivalidad de aquélla es más pronunciada debido a su descatable fusión nival y al 
mínimo invernal más marcado. Sin embargo, ambos contrastan fuertemente con el régimen registrado en los últimos 30 años (Figura 4.C), que a pesar de la influencia que puedan tener la explotación de los embalses, principalmente de Mediano y El Grado, el primero finalizado en 1960 (recrecido en 1974), el segundo en 1969, y que tiende a regularizar el hidrograma, muestra un carácter mucho más pluvial, habiendo casi desaparecido el mínimo invernal. Si la comparación la realizamos sobre la estimación de caudales en régimen natural (Figura 4.D), es decir, sin interferencia humana, realizada para el Plan Hidrológico de la cuenca del Ebro, apreciamos igualmente haberse producido un suavizado del mínimo invernal. En definitiva, se constata algo ya intuido y apuntado por otros autores: un cambio claro del régimen hidrológico en los últimos 120 años, atenúandose el componente nival desde finales del siglo XIX, pasando de regímenes nivopluviales (Figuras 4.A y 4.B) a pluvionivales (Figuras 4.C y 4.D), una vez avanzado el siglo XX.

Si atendemos a los valores numéricos de las aportaciones mensuales (Tabla 2), vemos cómo en los hidrogramas representados en las figuras 4.A, 1881-1894, y 4.B, 1928-1959, los meses de abril, mayo y junio concentran más del $40 \%$ de las aportaciones anuales, mientras que en la 4.D, 1940-1986, ha disminuido ya al $37 \%$ y en la 4.C, 1970-2001, apenas alcanzan el $32 \%$. Es destacable que mientras el mínimo estival de agosto se mantiene en todos los hidrogramas en el entorno del $4 \%$ respecto de la aportación total, el invernal ha pasado de no superar el 4\% en el hidrograma de la figura 4.A (1881-1894), a quedarse por encima del 5\% en el 4.B (1928-59), y del $7 \%$ en los otros dos.

En cuanto a los caudales y al volumen de las aportaciones, estos arrojan valores mucho mayores que las series consideradas actualmente como oficiales, es decir, desde 1913. La media interanual resultante de la serie 1881-1894 alcanza los 3.455 hm3/año, considerando sólo años completos, muy por encima de los $2.554 \mathrm{hm} 3 /$ año de la serie histórica oficial, aunque no tan lejos de los valores de la serie en régimen natural antedicha, cifrados en 2.915 hm3/año (Tabla 3 y 4 ).

Estas aportaciones singularmente elevadas no parecen excesivamente coherentes con los registros posteriores de la serie oficial de aforos, ni con la estimación en régimen natural. Es cierto que durante las últimas décadas del siglo XIX es constatable un periodo de mayor pluviosidad en el noreste de la Península Ibérica, pero este aumento de las precipitaciones no excede del 10\% sobre la lluvia media del periodo 1850-1950 (Saz Sánchez, 2003), mientras que las aportaciones medias según la serie 1881-1894 superan en un 35\% a la media histórica y en un $18 \%$ a la estimación en régimen natural, una estimación que, como hemos dicho, tiene en cuenta los consumos achacables al regadío y otros usos. Tampoco el cambio de sección efectuado en 1886 permite afirmar una mayor validez de la segunda parte de los datos pues el orden de magnitud permanece invariable. 

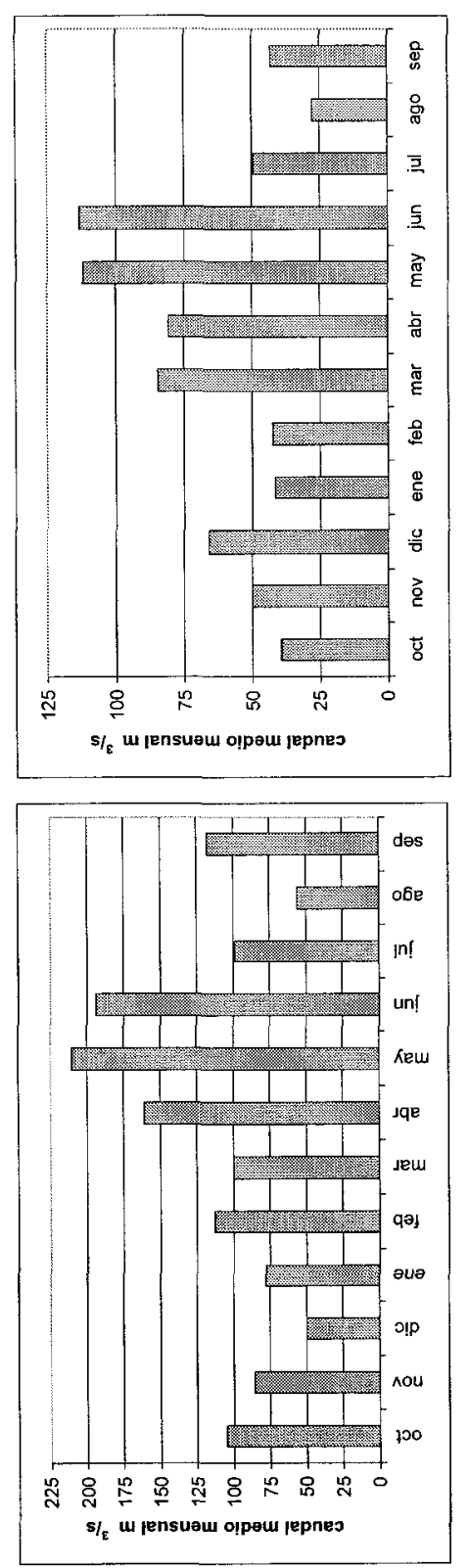

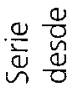

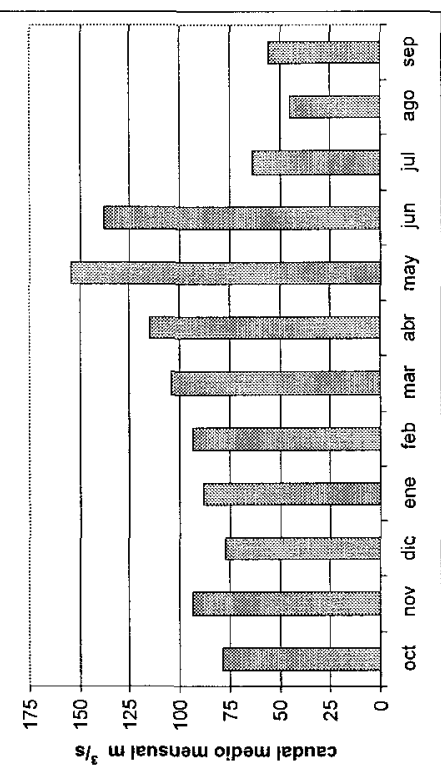

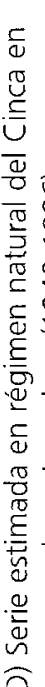

कิ

ᄃํ.

용

$\frac{\pi}{0} \frac{0}{0}$

过泣

$\bar{O} \frac{\mathrm{Q}}{\mathrm{O}}$

$\leadsto$ 号

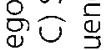

एक

용

过

ठे है ?

능

옹

응 完

迅

은 음 $\frac{\bar{\pi}}{}$

这

子小잉

엉으 은

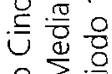

을 음 은

둬를

票

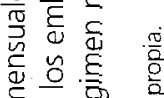

है

응 등

를

$\stackrel{\alpha}{\pi}$

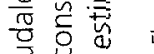

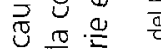

용

는

है

휸 흔

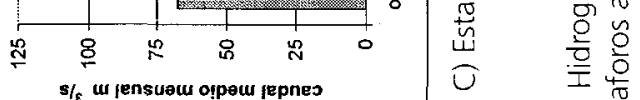

$\dot{\sigma}$

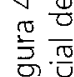

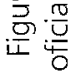


Tabla 2. Aportaciones mensuales y anuales de los hidrogramas correspondientes de la Figura 4 y sus pesos porcentuales.

\begin{tabular}{|lcccccccc|}
\hline & 4.A & 4.B & 4.C & 4.D & 4.A \% & 4.B \% & 4.C \% & 4.D \% \\
\hline oct & 281,7 & 105,8 & 180,9 & 210,3 & $7,84 \%$ & $5,36 \%$ & $7,35 \%$ & $7,23 \%$ \\
nov & 222,9 & 129,1 & 183,7 & 242,5 & $6,21 \%$ & $6,54 \%$ & $7,46 \%$ & $8,33 \%$ \\
dic & 135,2 & 176,6 & 190,0 & 206,6 & $3,77 \%$ & $8,95 \%$ & $7,72 \%$ & $7,10 \%$ \\
ene & 208,8 & 111,8 & 235,1 & 236,2 & $5,81 \%$ & $5,67 \%$ & $9,55 \%$ & $8,12 \%$ \\
feb & 273,0 & 103,1 & 224,1 & 225,9 & $7,60 \%$ & $5,22 \%$ & $9,10 \%$ & $7,76 \%$ \\
mar & 267,8 & 226,3 & 226,4 & 279,2 & $7,46 \%$ & $11,47 \%$ & $9,20 \%$ & $9,59 \%$ \\
abr & 418,2 & 209,0 & 206,0 & 298,6 & $11,64 \%$ & $10,59 \%$ & $8,37 \%$ & $10,26 \%$ \\
may & 562,0 & 300,6 & 293,8 & 415,8 & $15,65 \%$ & $15,23 \%$ & $11,93 \%$ & $14,29 \%$ \\
jun & 503,8 & 293,7 & 300,8 & 357,6 & $14,03 \%$ & $14,88 \%$ & $12,22 \%$ & $12,29 \%$ \\
jul & 264,0 & 132,0 & 174,7 & 170,4 & $7,35 \%$ & $6,69 \%$ & $7,10 \%$ & $5,86 \%$ \\
ago & 149,3 & 73,9 & 110,0 & 122,0 & $4,16 \%$ & $3,74 \%$ & $4,47 \%$ & $4,19 \%$ \\
sep & 305,1 & 111,9 & 136,6 & 145,0 & $8,49 \%$ & $5,67 \%$ & $5,55 \%$ & $4,98 \%$ \\
anual & 3591,8 & 1973,9 & 2462,1 & 2910,1 & $100,00 \%$ & $100,00 \%$ & $100,00 \%$ & $100,00 \%$ \\
\hline
\end{tabular}

Fuente: Confederación Hidrográfica del Ebro y elaboración propia.

Tabla 3. Caudales medios mensuales y anuales en $\mathrm{m}^{3} / \mathrm{s}$. Aportaciones anuales en $\mathrm{hm}^{3}$.

\begin{tabular}{|c|c|c|c|c|c|c|c|c|c|c|c|c|c|}
\hline \multicolumn{14}{|c|}{ Años hidrológicos } \\
\hline & $1880-81$ & $1881-82$ & $1882-83$ & $1883-84$ & $1884-85$ & $1885-86$ & $1889-9$ & $1890-91$ & $1891-92$ & $1892-93$ & $1893-94$ & $1894-95$ & $\begin{array}{l}\text { valore } \\
\text { medio }\end{array}$ \\
\hline oct & & 83,1 & 47,2 & 48,4 & 70,9 & 86,4 & & 33,4 & 326,1 & 101,0 & 137,0 & 118,4 & 105,2 \\
\hline nov & & 39,4 & 35,9 & 29,3 & 34,4 & 199,1 & & 33,3 & 199,6 & 182,3 & 58,3 & 48,4 & 86,0 \\
\hline dic & & 35,3 & 79,5 & 26,7 & 29,9 & 46,1 & & 29,5 & 110,5 & 79,0 & 44,0 & 24,4 & 50,5 \\
\hline ene & 277,6 & 34,6 & 39,8 & 25,2 & 62,6 & 100,0 & 61,3 & 20,5 & 121,6 & 65,4 & 48,8 & & 78,0 \\
\hline feb & 348,2 & 34,0 & 77,0 & 68,8 & 226,7 & 85,3 & 75,8 & 15,8 & 197,2 & 71,2 & 41,3 & & 112,8 \\
\hline $\operatorname{mar}$ & & 42,7 & 107,8 & 131,4 & 92,1 & 134,1 & 110,6 & 53,1 & 194,6 & 88,5 & 45,0 & & $100, \mathrm{C}$ \\
\hline abr & 355,4 & 74,9 & 161,8 & 239,6 & 92,3 & 194,3 & 158,8 & 51,1 & 221,0 & 72,2 & 153,1 & & 161,3 \\
\hline may & 238,1 & 167,4 & 304,3 & 281,2 & 184,1 & 350,9 & 186,5 & 97,0 & 218,6 & 92,3 & 187,5 & & $209, \varepsilon$ \\
\hline jun & 239,0 & 103,0 & 289,3 & 207,2 & 346,5 & 207,7 & 162,9 & 142,6 & 140,3 & 96,8 & 202,5 & & 194,4 \\
\hline jul & 176,1 & 85,1 & 125,8 & 120,1 & 133,1 & 94,8 & 53,4 & 66,8 & 70,1 & 51,6 & 107,3 & & 98,6 \\
\hline ago & & 39,9 & 47,6 & 42,7 & 108,1 & 34,8 & & 32,2 & 90,2 & 42,1 & 63,8 & & 55,7 \\
\hline $\begin{array}{l}\text { sep } \\
\text { valores }\end{array}$ & 43.5 & 108,9 & 39,9 & 458,0 & 88,8 & 76,2 & 105,0 & 169,2 & 34,3 & 142,1 & 29,0 & & 117, \\
\hline $\begin{array}{l}\text { medios } \\
\text { aporta- }\end{array}$ & & 70,7 & 113,0 & 139,9 & 122,5 & 134,2 & & 62,0 & 160,3 & 90,4 & 93,1 & & 109,6 \\
\hline ciones & & $2.229,5$ & $3.563,7$ & $4.411,5$ & $3.862,0$ & $4.230,6$ & & $1.956,7$ & $5.056,7$ & $2.850,0$ & $2.937,0$ & & 3.455 \\
\hline
\end{tabular}

Fuente: Elaboración propia. 
Tabla 4. Aportaciones medias interanuales, máximas y mínimas en $\mathrm{hm}^{3}$.

\begin{tabular}{|lcccc|}
\hline \multicolumn{1}{|c}{ Denominación } & Periodo & Media & Máxima & Mínima \\
\hline Cinca en Ex- Monasterio de Escarpe & $1881-1894$ & 3.455 & 5.057 & 1.957 \\
Cinca en Fraga (EA 017) & $1928-1959$ & 1.972 & 3.180 & 483 \\
Cinca en Fraga (EA 017) & $1970-2001$ & 2.466 & 4.221 & 821 \\
Cinca en Fraga (EA 017) & $1928-2001$ & 2.554 & 4.746 & 483 \\
Cinca en desembocadura (estimación en & $1940-1986$ & 2.915 & 5.209 & 928 \\
régimen natural) & & & & \\
\hline
\end{tabular}

Fuente: Confederación Hidrográfica del Ebro y elaboración propia.

Lamentablemente, esta circunstancia, añadida a las cautelas con las que ya nos veníamos proveyendo, nos conduce a considerar como poco fiables en cuanto a su precisión volumétrica los datos aportados por la serie de aforos del Cinca en el exmonasterio de Escarpe, aunque tampoco pueda afirmarse rotundamente su invalidez.

Estos condicionantes también limitan uno de los análisis más interesantes de este tipo de datos: los caudales mínimos registrados en un momento del río de cuasi naturalidad y que podrían ser un buen elemento de referencia y contraste a la hora de la implantación de caudales ecológicos.

A pesar de nuestra creencia en una cierta sobreestimación de los caudales aforados, podría pensarse que, teniendo en cuenta los procedimientos de calibrado y preparación de las curvas de gasto de una sección de aforos, la curva estuviera bien calibrada para los caudales bajos y sólo los altos fueran erróneos, pero se trata de una suposición sin mayor fundamento. Con todo, aunque despreciemos los valores absolutos, su entidad relativa sigue teniendo cierto interés como elemento de referencia.

Ya hemos indicado que la serie de datos muestra diferencias de medición entre sus partes según la sección de control de aforo utilizada. Si atendemos a la primera, el caudal mínimo registrado es de $24,4 \mathrm{~m}^{3} / \mathrm{s}$; si atendemos a la segunda resulta de $11,8 \mathrm{~m}^{3} / \mathrm{s}$ (tabla 5). Es probable que la medición más correcta sea la segunda, suponiendo en buena lógica que el cambio de sección introduciría mejoras en el proceso, pero tampoco puede asegurarse.

Si evaluamos su valor relativo resulta que para el primer caso tenemos que el caudal mínimo de la primera sección, serie 1881-86, convertido en aportación anual, representa el 22,3\% de la aportación media de la serie completa, 1881-1894; para el caudal mínimo de la segunda sección, serie 1889-1894, resulta ser el 10,8\%. Se trata de unas referencias que pueden ser interesantes a la hora de contrastar futuras propuestas de caudales ecológicos. En estos momentos un porcentaje del 10\% es el que contempla con carácter general y de forma provisional el Plan Hidrológico de la 
Tabla 5. Caudales máximos y mínimos diarios en $\mathrm{m}^{3} / \mathrm{s}$.

\begin{tabular}{|lcccccccccccc|}
\hline \multicolumn{10}{|c|}{ Años hidrológicos } \\
\cline { 2 - 13 } & $\mathbf{1 8 8 0 - 8 1}$ & $\mathbf{1 8 8 1 - 8 2}$ & $\mathbf{1 8 8 2 - 8 3}$ & $\mathbf{1 8 8 3 - 8 4}$ & $\mathbf{1 8 8 4}-\mathbf{8 5}$ & $\mathbf{8 8 5 5 - 8 6}$ & $\mathbf{1 8 8 9 - 9}$ & $\mathbf{1 8 9 0 - 9 1}$ & $\mathbf{1 8 9 1 - 9 2} \mathbf{1 8 9 2 - 9 3}$ & $\mathbf{1 8 9 3 - 9 4} \mathbf{1 8 9 4}-\mathbf{9 5}$ \\
\hline Máx. & $1.334,5$ & 821,5 & $1.334,5$ & $2.997,8$ & $1.050,4$ & $1.519,3$ & $1.283,3$ & $2.889,6$ & $2.950,1$ & $1.148,8$ & 912,7 & 882,7 \\
Min. & 29,0 & 32,7 & 28,9 & 24,4 & 26,0 & 27,7 & 13,4 & 13,1 & 13,8 & 20,9 & 14,8 & 11,8 \\
\hline
\end{tabular}

Fuente: Elaboración propia.

cuenca del Ebro para todos los ríos de la cuenca, pudiéndose rebajar al 5\% para ríos de elevado caudal como el Cinca, lo que significa una cifra de $4,53 \mathrm{~m}^{3} / \mathrm{s}$.

Obviamente estas referencias son muy matizables puesto que el régimen hidrológico natural de entonces no es el actual (ni el modificado por las actividades humanas ni el restituido al régimen natural) y que los datos analizados, al margen de su, cuando menos, no contrastable precisión, corresponden a un periodo calificado como húmedo. Son numerosos los registros inferiores a $10 \mathrm{~m}^{3} / \mathrm{s}$ durante los periodos secos constatables en la serie oficial de aforos de la estación 17 Cinca en Fraga, incluso antes de la construcción de los grandes embalses y del elevado desarrollo del regadío.

En otro orden, los caudales máximos registrados resultan sorprendentes. Nos encontramos con avenidas con caudal cercano a $3.000 \mathrm{~m}^{3} / \mathrm{s}$ (tabla 5), frente a los valores conocidos en las dos avenidas mayores del s.XX: en $1937 \mathrm{con} 2.600 \mathrm{~m}^{3} / \mathrm{s}$ en Fraga, y 1982 con $2.100 \mathrm{~m}^{3} / \mathrm{s}$ en idéntico lugar, aunque en este último caso sin duda atenuada por la laminación que ya pudieron aportar los embalses. Sin embargo, a falta de una comprobación más exhaustiva, no hay noticias de inundaciones de envergadura en aquellas fechas, tan sólo en septiembre de 1884 y con alcance limitado. De nuevo, debemos concluir que se trata de registros de escasa validez.

\section{Conclusiones}

En definitiva, aunque no con la precisión que nos gustaría, los datos disponibles de caudales del Cinca en el siglo XIX nos facultan para acercarnos a sus características hidrológicas en aquella época. En este sentido se trata de una información inestimable por cuanto nos permite conocer la evolución de los patrones hidrológicos del río, obviamente enlazados con los climáticos, y de dotarnos con algunos elementos comparativos respecto a la situación actual, especialmente relevante por lo que puede emerger en la comparación de aquel régimen fluvial con uno modificado por los intensos usos humanos, y que podría ser susceptible de un mayor estudio. 
En particular podemos concluir lo siguiente:

-La medición sistemática de caudales por parte de la Administración Hidráulica comenzó en la cuenca del Ebro en fechas tan tempranas como 1881, aunque las series de caudales consideradas actualmente como oficiales comiencen en 1913.

-La serie del Cinca en desembocadura que recoge el Proyecto de Riegos del Alto Aragón de 1911 para el periodo1881-1894 es consistente, pero incurre aparentemente en falta de precisión de medida y en conjunto en una sobreestimación de los caudales aforados.

-Con todo, esta serie nos permite observar el régimen hidrológico del Cinca en las condiciones más próximas a las naturales conocidas, y confirmar la pérdida de una gran parte del comportamiento nival que tenía a finales de siglo XIX.

\section{Agradecimientos}

A Miguel Ángel García Vera (Confederación Hidrográfica del Ebro) y Javier del Valle Melendo (Departamento de Geografía, Universidad de Zaragoza), por la revisión crítica del texto.

\section{Bibliografía}

Confederación Hidrográfica del Ebro (2000) Los aprovechamientos en la cuenca del Ebro. Afección al régimen bidrológico fluvial. Director: García Vera, M.A. Zaragoza. Inédito.

Escuer, J.L. (2004) El Cinca a Fraga: una reflexió sobre dinàmica fuvial $i$ activitat bumana. Instituto de Estudios del Bajo Cinca.

Fanlo Loras, A. (1996) Las Confederaciones Hidrográficas $y$ otras administraciones bidrâulicas. Madrid. Civitas.

Frutos, M.L., Ollero, A. y Sánchez-Fabre, M., (2004) Caracterización del río Ebro y su cuenca y variaciones en su comportamiento hidrológico. En Gil Olcina, A. (coord.) Alteración de los regímenes fluviales peninsulares, Murcia, Instituto Euromediterráneo de Hidrotecnia y Fundación Caja Murcia, 233-280.

García Ruiz, J.M., Beguería, S., López, J.I., Lorente, A. y Seeger, M. (2001) Los recursos bidricos superficiales del Pirineo aragonés y su evolución reciente. Geoforma. Logroño.

Mateu Bellés, J.F.(2004) Los aforos de los ríos peninsulares durante la primera etapa de las divisiones hidrológicas (1865-1876). En 
Historia, clima y paisaje: estudios geogräficos en memoria del profesor Antonio López Gómez, 363-382, Valencia.

Ríos (de los) Martín, F., Nicolau Sabater, J. (1911) Proyecto de Riegos del Alto Aragón. Barcelona.

Servicio Central Hidráulico Instrucciones sobre aforos. Madrid.

(1911)

Servicio Central Hidráulico (1913). Aforos. Régimen de los ríos de España en el año 1912. Madrid.
Saz Sánchez, M.A. (2003). Temperaturas y precipitaciones on la mitad norte de España desde el siglo XV. Estudio dendroclimático. Consejo de Protección de la Naturaleza de Aragón. Zaragoza.

Valle (del) Melendo, J. (1997) La precipitación media anual en el sector alto de la cuenca del Cinca. Pirineos, 149-150, 121-144.

Valle (del) Melendo, J. (coord) (2004) Análisis territorial de la comarca del Sobrarbe. Sobrarbe $n^{\circ} 9$ (monográfico). 